\title{
Importancia de las técnicas de Respiración Cardiopulmonar Básica. Un estudio en la Región de Murcia Importance of basic CPR techniques. Astudy in the Region of Murcia
}

\begin{abstract}
J.ArturoAbraldes Valeiras
Universidad de Murcia

Resumen: Anualmente en España mueren un gran número de personas por enfermedades relacionadas con el corazón. El paro cardíaco es la causa principal en este tipo de muertes. Conocer y dominar las técnicas de Reanimación Cardiopulmonar Básica (RCPB) son fundamentales para auxiliar a un accidentado de estas características. En este estudio se analizó 1) la importancia de conocer estas técnicas en la población en general, y 2) la percepción de la dificultad de comprensión y ejecución de las técnicas. Se utilizó un cuestionario diseñado «ad hoc» como instrumento para la recogida de información. El instrumento se sometió a procesos de validación y fiabilidad para el estudio. Se administró el cuestionario a 235 personas voluntarias de edades comprendidas entre los 10 y los 65 años. Se realizó un análisis estadístico descriptivo, en función del género y de las variables importancia de conocimiento y aprendizaje / ejecución de las técnicas. Entre los resultados más relevantes, podemos destacar un interés de la sociedad a aumentar la formación en este tipo de conocimientos. Formación que sería ideal en la mayoría de los grupos de edad de la población (adolescentes a ancianos). Así mismo, las técnicas de RCPB son fáciles de comprender y, en menor medida de ejecutar, percepción más relevante entre de la gente que manifiesta conocer y dominar dichas técnicas.
\end{abstract}

Palabra clave: Soporte vital básico, enfermedad cardiovascular, primeros auxilios, educación.

Abstract: Annually in Spain many people die from diseases related to heart. Heart attack is the main cause of such deaths. Know and control the basic techniques of basic Cardiopulmonary resuscitation (CPR) are essential to help a person away from these features. This study examined 1) the importance of knowing these techniques in the general population, and 2) the perception of the difficulty of understanding and application of techniques. We used a questionnaire designed «ad hoc» as a tool for collecting information. The instrument was subjected to validation and reliability for the study. The questionnaire was completed by 235 volunteers aged between 10 and 65 . We performed a descriptive analysis, based on gender and the variables importance of knowledge and learning / apply techniques. Among the most relevant results, we emphasize an interest of society to improve training in this type of knowledge. Training would be ideal in most age groups of people (teens to seniors). Likewise, CPR techniques are easy to understand and execute a lesser extent, relevant perception among people who acknowledge and application these techniques.

Key words: Basic Life Support, cardiovascular disease, first aids, education.

\section{Introducción}

Cada año, casi 40.000 personas mueren en España debido a enfermedades cardiovasculares. En el año 2004 éstas fueron la principal causa de muerte, representando el 33,3\% del total de defunciones según los datos ofrecidos por el Instituto Nacional de Estadística (INE). Dentro de este grupo las enfermedades isquémicas del corazón (infarto agudo de miocardio, angina de pecho, etc.) fueron la primera causa de muerte (INE, 2006).

Las personas más propensas a padecer un paro cardiaco son varones, próximos a los 60 años y mujeres cercanas a los 70 años de edad. Así mismo, las personas obesas tienen mayor probabilidad de sufrir una angina de pecho. De mismo modo, los hombres presentan el doble de probabilidad que las mujeres de sufrir un paro cardiaco (ERC, 2005).

Aproximadamente, el 50\% de estas muertes se produce antes de que la víctima pueda llegar al hospital. Las estadísticas nos indican que en el 75\% de los paros cardiacos ocurren en el hogar (ERC, 2005). El efecto de estos trastornos circulatorios es la anoxia, que desemboca en daños irreversibles a nivel neurológico o la muerte en algunos minutos (Liberman, Golberg, Mulder y Sampalis, 2000). Para evitar estos efectos se hace extremadamente necesaria una intervención rápida y eficaz, ya que tan sólo se dispone de 3 a 5 minutos para realizar una primera intervención con técnicas de Reanimación Cardiopulmonar Básica (RCPB). Este tiempo aumenta a 15 minutos cuando el soporte vital avanzado se realiza con ayuda de desfibrilador, por parte del personal médico especializado (Pearn, 2000; Uray et al., 2003).

Teniendo en cuenta que los servicios de emergencia urbanos, incluso bien organizados, tardan como mínimo 10 minutos en llegar al lugar del

Fecha recepción: 07-11-10 - Fecha envío revisores: 16-11-10 - Fecha de aceptación: 17-12-10 Correspondencia: J. Arturo Abraldes Valeiras

Calle Argentina, s/n

30720 - Santiago de la Ribera. Murcia

E-mail: abraldes@um.es accidente (Cummins, Ornato, Thies y Pepe, 1991; Eisenburger y Safar, 1999) es de vital importancia la presencia de personas con conocimientos en RCPB en el mismo lugar. En el $80 \%$ de las muertes súbitas por paro cardiaco, éstas se producen en presencia de un familiar o un amigo (Abraldes, Rodríguez, Rubio y Martínez, 2006) y miembros del público en general, lo que justifica la necesidad de que la mayor cantidad de personas posean conocimientos en RCPB (Celenza et al., 2002; Liberman, Golberg, Mulder y Sampalis, 2000; Morgan, Donnelly, Lester y Assar, 1996; Sekimoto et al., 2001; Selby et al., 1982; Uray et al., 2003). La rápida intervención en un paro cardiaco mediante técnicas de RCPB duplica las posibilidades de supervivencia de la víctima (Abraldes, Rodríguez, Rubio y Martínez, 2006; ERC, 2005).

De entre el público en general son especialmente las mujeres las que tienen mayor interés en los aspectos de salud pública, las que más llaman a los servicios de información sanitaria, y además, las más interesadas en recibir información y formación en la realización de las técnicas de RCPB (Selby et al., 1982). Un aspecto importante para que las personas realicen cursos de primeros auxilios y en especial de RCPB, reside en la motivación de la persona por aprender estas habilidades y la importancia que ellos les dan a estos conocimientos (Axelsson, Herlitz y Fridlund, 2000). Del mismo modo, dependiendo del trabajo y/o actividad realizada por cada persona deberá tener un mayor conocimiento de estas técnicas por su probabilidad de aparición (deportistas, bomberos, educadores, etc.) (Abraldes y Ortín, 2008; Ortín y Abraldes, 2007).

Por tanto, se planteó en este estudio un doble objetivo. Por un lado, analizar la importancia de conocer las técnicas de RCPB en la población en general, y la percepción de la dificultad de comprensión y ejecución de las mismas. Se observaron estas variables en relación al género de la muestra, de tal forma que se pudo conocer la motivación y el grado de comprensión y dificultad práctica que suponen los cursos específicos para la población en general. 


\section{Método}

\subsection{Muestra}

La muestra del estudio queda delimitada a personas residentes en la Región de Murcia (España). Ésta asciende a un total de 235 personas, de entre los que han contestado a la pregunta de género $(\mathrm{n}=224)$ el $38,39 \%(n=86)$ son hombres y el $61,61 \%(n=138)$ son mujeres. Los participantes presentan una edad media total de 30,85 años $( \pm 12,91)$, siendo la población femenina más joven que la masculina (29,05 años ( \pm $13,41)$ de media en las mujeres, frente a los 33,99 años $( \pm 11,53)$ de término medio en los hombres). Así mismo, manifiestan que el 64,73\% de ellos realiza actividad física de forma frecuente (más de un día por semana).

\subsection{Procedimiento}

La elección del cuestionario como instrumento de esta investigación atendió a ventajas que éstos presentan (Gómez, 1992; Fernández Balboa, 1997) y que, fundamentalmente, se centran, en dos aspectos: la ventaja de recoger información a grandes muestras y, la brevedad de tiempo en la que la información es recogida. Por las características que se reflejan en este estudio nos hemos decantado por este instrumento, a pesar de los inconvenientes que también presenta (Moreira, 2004; Magalhães y Hill, 2005)

El planteamiento de los ítems del cuestionario y de las áreas relacionadas a sus contenidos se estableció en función de las hipótesis planteadas, así como de los objetivos propuestos en la investigación. Se diseñó un primer cuestionario estructurado teniendo en cuenta las diferentes áreas temáticas que se deseaba investigar. Una vez diseñado este primer cuestionario, se sometió a las observaciones y aportaciones de otros investigadores, que llevaron a una mejor redacción del mismo y a la sustitución y/o inclusión de nuevos ítems de relevancia.

La valoración y validación de la herramienta se realizó por consenso por parte de doctores y expertos afines a las áreas incluidas en el cuestionario. Estas áreas estaban representadas por formadores en primeros auxilios, estadísticos, médicos y psicólogos. Dichas aportaciones llevaron a otra modificación del cuestionario, dotándole de un mejor lenguaje y una mayor claridad de contenidos a la hora de rellenarlo.

Finalmente se llegó a la redacción de un cuestionario, que contenía un total de 24 preguntas específicas ( 3 abiertas, 18 cerradas y 3 semicerradas). A su vez, las preguntas cerradas disponían de, según los casos, otras preguntas afines al contenido de la pregunta principal o una opción («otros») para aclarar o completar la respuesta.

A su vez, y para una mejor comprensión del mismo, éste se dividía en cuatro grandes apartados o contenidos:

.En el primer apartado se encuentran las preguntas de control, las cuales se referían a la comunidad en la que residían, el género del encuestado, profesión, fecha de nacimiento y su práctica de actividad física habitual. Éstas eran un total de 5, de ellas 3 eran de carácter abierto y 2 de carácter cerrado.

-Un segundo apartado se centra principalmente en preguntas sobre el conocimiento que los encuestados tenían sobre las técnicas de RCP. Aquí, nos encontramos con un total de 8 preguntas, de las cuales 6 son de carácter cerrado y 2 de carácter semicerrado (cerradas con posibilidad de una respuesta abierta).

- Un tercer apartado incluye preguntas de carácter sociodemográfico y de valoración de un taller de RCP. En éste nos encontramos con 6 preguntas, de las cuales 5 eran cerradas y 1 semicerrada.

- Un cuarto y último apartado se centra en preguntas sobre la importancia de las técnicas de RCP. Aquí nos encontramos con un total de 5 preguntas, todas ellas de carácter cerrado.

Para detectar la fiabilidad se procedió a la administración del mismo cuestionario dos veces, a varios sujetos, con una semana de diferencia temporal entre una prueba y otra. Dicha fiabilidad se realizó a un grupo de sujetos que pertenecían a la población de estudio pero que no estaban incluidos en la muestra (Magalhães y Hill, 2005). Obteniéndose valores de fiabilidad por encima de 0,86 en el índice de Kappa.

\subsection{Diseño}

Para la realización de este estudio, se llevó a caboun diseño transversal descriptivo analizando los valores de las variables recogidas en relación al género de los sujetos entrevistados (adaptado de Hernández, Fernández, \& Baptista, 2000). La recogida de datos se llevó a cabo al mismo tiempo que se celebraba la Semana de la Ciencia y la Tecnología de la Región de Murcia. En ella, se ofertaba una práctica de primeros auxilios donde se demostraban las técnicas de RCPB. Los cuestionarios eran rellenados voluntariamente, tanto por la gente que participaba en el taller como por espectadores que solamente observaban las demostraciones.

La introducción de datos se realizó a través de una plantilla diseñada, para tal fin, con el programa Excel del paquete informático Microsoft Office 2003 del entorno Windows XP Profesional, codificando y categorizando cada una de las variables. Una vez introducidos los datos, se realizaba una revisión de los mismos de forma aleatoria, escogiendo diferentes cuestionarios al azar y se comprobaba si se habían cometido errores en la trascripción al ordenador. En total se revisaron aleatoriamente un total de 120 cuestionarios (51,06\% del total).

\subsection{Estadística}

El análisis estadístico de los datos se realizó con el paquete informático SPSS 15.0 en el entorno Windows XP Profesional, llevándose a cabo un análisis descriptivo de los datos (frecuencias, absolutas, medias, porcentajes, desviaciones, ...). Se realizaron tablas de contingencia en función de las variables de estudio y el sexo.

\section{Resultados}

Los principales resultados encontrados tras el análisis de datos se detallan en las siguientes tablas que hacen referenciaal interés, importancia y percepción de las técnicas de RCPB en la muestra de estudio.

\begin{tabular}{|l|c|c|c|}
\hline \multicolumn{5}{|c|}{ Tabla 1. Interés de los encuestados en ampliar su formación en las técnicas de RCPB } \\
$(\mathrm{N}=220)$. \\
\hline Género & Casos & No & Sí \\
\hline \multirow{2}{*}{ Masculino } & $\mathrm{n}$ & 16 & 67 \\
\cline { 2 - 4 } & $\%$ & 19,28 & 80,72 \\
\hline \multirow{3}{*}{ Femenino } & $\mathrm{n}$ & 26 & 111 \\
\cline { 2 - 4 } & $\%$ & 18,98 & 81,02 \\
\hline \multirow{3}{*}{ Total } & $\mathrm{n}$ & 42 & 178 \\
\cline { 2 - 4 } & $\%$ & 19,09 & 80,91 \\
\hline \multirow{2}{*}{$\begin{array}{l}\text { Leyenda: (N) Número total de sujetos que responden a la pregunta (No) No tiene } \\
\text { interés en formarse en contenidos de RCP. (Si) Presenta interés en formarse en } \\
\text { contenidos de RCP. }\end{array}$} \\
\hline
\end{tabular}

\begin{tabular}{|c|c|c|c|c|c|}
\hline \multicolumn{6}{|c|}{ Tabla 2. Importancia del conocimiento de la RCP en función del rango de edad. } \\
\hline \multirow{2}{*}{ Género } & Casos & Niños & Adolescentes & Adultos & Mayores \\
\hline & $\mathrm{N}$ & 170 & 209 & 202 & 179 \\
\hline \multirow{2}{*}{ Masculino } & $\mathrm{n}$ & 42 & 72 & 74 & 62 \\
\cline { 2 - 6 } & $\%$ & 68,9 & 94,7 & 98,7 & 95,4 \\
\hline \multirow{3}{*}{ Femenino } & $\mathrm{n}$ & 85 & 129 & 123 & 107 \\
\cline { 2 - 6 } & $\%$ & 81,0 & 99,2 & 100,0 & 96,4 \\
\hline \multirow{2}{*}{ Total } & $\mathrm{n}$ & 127 & 201 & 197 & 169 \\
\cline { 2 - 6 } & $\%$ & 76,5 & 97,6 & 99,5 & 96,0 \\
\hline $\begin{array}{l}\text { Leyenda: (N) Número total de sujetos que responden a la pregunta. (Niños) } \\
\text { Importancia de que los niños sí tengan conocimiento de la RCP. (Adolescentes) } \\
\text { Importancia de que los adolescentes sí tengan conocimiento de la RCP. (Adultos) } \\
\text { Importancia de que los adultos sí tengan conocimiento de la RCP. (Mayores) } \\
\text { Importancia de que los mayores sí tengan conocimiento de la RCP. }\end{array}$ \\
\hline
\end{tabular}




\begin{tabular}{|c|c|c|c|c|}
\hline \multicolumn{5}{|c|}{ Tabla 3. Importancia de los contenidos en RCP en la formación escolar. } \\
\hline Género & Casos & Colegios & Institutos & Ambos \\
\hline & $\mathrm{N}$ & 212 & 206 & 235 \\
\hline \multirow{2}{*}{ Masculino } & $\mathrm{n}$ & 70 & 75 & 58 \\
\cline { 2 - 5 } & $\%$ & 89,7 & 98,7 & 65,9 \\
\hline \multirow{3}{*}{ Femenino } & $\mathrm{n}$ & 117 & 124 & 102 \\
\cline { 2 - 5 } & $\%$ & 90,7 & 97,6 & 71,8 \\
\hline \multirow{3}{*}{ Total } & $\mathrm{n}$ & 187 & 199 & 160 \\
\cline { 2 - 5 } & $\%$ & 90,3 & 98,0 & 69,6 \\
\hline \multirow{5}{*}{$\begin{array}{l}\text { Leyenda: }(N) \text { Número total de sujetos que responden a la preg unta. (Colegios) Si } \\
\text { considera importante el aprendizaje de los contenidos de la RCP en el colegio. } \\
\text { (Institutos) Sí considera importante el aprendizaje de los contenidos de la RCP en el } \\
\text { instituto. (Ambos) Sí considera importante el aprendizaje de los contenidos de la } \\
\text { RCP tanto en colegios como institutos }\end{array}$} \\
\hline
\end{tabular}

\begin{tabular}{|} 
Tabla 4. Percepción de la facilidad de aprendizaje y ejecución de RCP en función del \\
interés (N = 225).
\end{tabular}

\begin{tabular}{|c|c|c|c|c|c|}
\hline \multicolumn{6}{|c|}{$\begin{array}{c}\text { Tabla 5. Percepción de la facilidad de aprendizaje y ejecución de las técnicas de RCP en } \\
\text { función de si saben o no realizar las técnicas de RCP (N =231). }\end{array}$} \\
\hline \multirow{2}{*}{ Género } & Casos & \multicolumn{2}{|c|}{ No saben realizar RCPB } & \multicolumn{2}{|c|}{ Sí saben realizar RCPB } \\
\hline & & \multicolumn{2}{|c|}{$120(51,9 \%)$} & \multicolumn{2}{|c|}{$111(48,1 \%)$} \\
\hline & & Aprender & Realizar & Aprender & Realizar \\
\hline \multirow{3}{*}{ Femenino } & $\mathrm{n}$ & 35 & 15 & 36 & 26 \\
\cline { 2 - 7 } & $\%$ & 85,37 & 42,86 & 90,00 & 68,42 \\
\hline \multirow{3}{*}{ Masculino } & $\mathrm{n}$ & 61 & 28 & 63 & 32 \\
\cline { 2 - 6 } & $\%$ & 88,41 & 43,75 & 95,45 & 54,24 \\
\hline \multirow{3}{*}{ Total } & $\mathrm{n}$ & 96 & 43 & 99 & 58 \\
\cline { 2 - 6 } & $\%$ & 87,27 & 43,43 & 93,40 & 59,79 \\
\hline
\end{tabular}

Leyenda: (N) Número total de sujetos que responden a la pregunta. (Aprender) Facilidad de dominar conceptualmente las técnicas de RCP. (Realizar) Facilidad de ejecución correcta de las técnicas de $R C P$.

\section{Discusión}

La gran mayoría de los sujetos encuestados $(80,91 \%)$ indican estar interesados en ampliar su formación en RCPB (Tabla 1), no existiendo apenas diferencias entre el porcentaje de hombres $(80,72 \%)$ y el de mujeres $(81,02 \%)$. Estos datos, en principio discrepan con el estudio de Selby et al (1982) que expresa un mayor interés por parte de las mujeres con respecto al interés en la formación de estos contenidos. Sin embargo, hay que tener en cuenta que en valores absolutos, de los 178 sujetos que respondieron a esta pregunta afirmativamente son casi el doble de mujeres (111) que de hombres (67).

En relación a la importancia (Tabla 2) que los encuestados dan a quienes deberían conocer y dominar las técnicas de RCPB en función de la franja de edad, el $97,6 \%$ manifiestan que deberían ser conocidos por adolescentes (entre 12 y 18 años), el 99,5\% por adultos (entre 18 y 65 años) y el $96 \%$ por personas mayores de 65 años. A este respecto, apreciamos que en más del 96\%, se manifiesta que los mayores de 12 años deberían conocer y dominar este contenido. Así mismo, vemos como estos contenidos ya se imparten en formaciones básicas y regladas, como el carnet de conducir, cursos de monitores y otros, en los que mayoritariamente sus alumnos presentan una edad entorno a los 16 años.

Sin embargo, el porcentaje disminuye cuando se trata de niños menores de 12 años (76,5\%), quizás por la percepción errónea de que, en esas edades no pueden ayudar ante un accidente de tal envergadura. En contraposición a esto, existe evidencia científica de que niños de edades entre los 6 y 15 años son capaces de comprender y practicar adecuadamente los primeros auxilios, incluyendo las técnicas de RCPB (Frederick, Bixby, Orzel, Stewart-Brown y Willett, 2002; Lewis, Fulstow y Smith, 1997; Uray et al., 2003). Es notorio como, en este aspecto, las mujeres manifiestan en mayor porcentaje $(81,0 \%)$ frente a los hombres $(68,9 \%)$, la importancia de adquirir estos conocimientos en los menores de 12 años. Aspecto que concuerda con los estudios aportados por Selby et al (1982) y Lewis, Fulstow y Smith, (1997).

No obstante, al hablar de si estos contenidos deberían incluirse dentro de la formación de los escolares de primaria y secundaria (Tabla 3 ), la gran mayoría indica que sería de gran importancia incluirlos en colegios $(90,3 \%)$ o institutos $(98,0 \%)$, coincidiendo estas cifras con el estudio de Uray et al. (2003), en el que el $94 \%$ de los sujetos encuestados (padres de alumnos) indicaron que debería ser una asignatura obligatoria en los colegios con una carga lectiva de una hora al mes. Así mismo, hemos encontrado propuestas de intervención de estos contenidos en escuelas que muestran resultados muy satisfactorios en el aprendizaje (Lewis, Fulstow y Smith, 1997). Una línea adecuada de enfoque de estos contenidos en secundaria sería la llevada acabo en Nueva Zelanda donde desde 1999 en el Currículo de Educación Física y Salud para las escuelas se introdujo la opción de tratar los contenidos en materia de RCPB en las edades de entre 5 y 18 años (Lafferty, Larsen y Galletly, 2003).

Abraldes y Ortín (2008) estudiaron el conocimiento en los primeros auxilios en profesores de Educación Física de enseñanza secundaria. Las conclusiones manifestaban un bajo nivel de formación general, tanto en hombres como en mujeres, y sobre todo en contenidos de RCPB, frente a otros como traumatismos o heridas. Datos similares a los encontrados en otros estudios referentes a la enseñanza primaria (García, Solís y Vasques, 1998). Lo que nos lleva a cuestionar resultados notables (Frederick, Bixby, Orzel, Stewart-Brown y Willett, 2002; Lewis, Fulstow y Smith, 1997; Uray et al., 2003) en estos contenidos en los alumnos de educación primaria y secundaria, si los profesores no están debidamente actualizados (Ortín y Abraldes, 2007).

Otro hecho a destacar es la apreciación que tiene el público en general sobre la facilidad de aprendizaje y realización de las técnicas de RCPB (Tabla 4). La mayoría de los encuestados creen que estas técnicas son fáciles de dominar conceptualmente, tanto interesados $(90,06 \%)$ o no en estos contenidos $(89,74 \%)$. Sin embargo, a la hora de realizarlos en la práctica, el grado de percepción de facilidad disminuye a un $50 \%$ en aquellos que no tienen interés en éstos contenidos y, de manera similar, a un 52,29\% en aquellos que sí tienen interés en este tipo contenidos.

Por otro lado, si comparamos valores teniendo en cuenta la variable de saber realizar las técnicas de RCPB, prácticamente no se observan diferencias entre los que no saben realizar la RCPB $(87,27 \%)$ y los que saben realizarla $(93,40 \%)$ en cuanto a su percepción de facilidad de aprendizaje.

La percepción de la facilidad de ejecución de las técnicas de RCPB (Tabla 5) se ve incrementada en los sujetos que saben realizar la RCPB $(59,79 \%)$ con respecto a los que no la saben realizar $(43,43 \%)$. Este aspecto parece indicar que las personas que saben realizar la RCPB perciben más facilidad en la ejecución de las técnicas de RCPB que los que no conocen previamente estas técnicas. Los datos encontrados muestran mayores valores porcentuales entre las personas que tienen un mayor interés y conocimiento, sobre la facilidad de comprensión y ejecución de las técnicas de RCPB. Estos datos están en relación con la 
importancia que tiene el aprendizaje en función de la motivación que tiene el alumno en este tipo de contenidos (Axelsson, Herlitz y Fridlund, 2000). Este aspecto nos muestra una vía de intervención en la que se planteen cursos de formación para aquellas personas que deseen formarse en este campo. A este respecto, los organismos públicos y asociaciones encargadas de la formación y divulgación de estos contenidos, deberían aunar esfuerzos en informar a la sociedad de la importancia de los mismos. Así, se vería aumentada la motivación y, en consecuencia, el conocimiento y aprendizaje de dichos contenidos.

\section{Conclusiones}

Como conclusiones de este estudio podemos afirmar las siguientes: -Encontramos un interés similar en relación al género, en el aprendizaje de las técnicas de RCPB.

-Existe la mayoritaria opinión de que esta formación debería ser conocida por toda la sociedad. Las mujeres manifiestan más que los hombres, que incluso ésta la debería de conocer los niños menores de 12 años.

- La formación en estos contenidos debería incluirse en las escuelas, tanto en primaria (colegios) como en secundaria (institutos), lo que viene a reforzar la formación en grupos de edad joven.

- La percepción encontrada para el aprendizaje de estas técnicas indica que es asequible a la inmensa mayoría, principalmente a nivel de comprensión (aprender como se hace) y, en menor medida, a nivel de ejecución práctica.

- La percepción de facilidad de aprendizaje de estas técnicas es mayoren aquellas personas que presentan interés en estos contenidos, asícomo en aquellas que manifiestan dominar las técnicas de RCPB.

\section{Referencias}

Abraldes, JA. y Ortín, A. (2008). La formación del profesorado de Educación Física en Primeros Auxilios. Almería: Asociación de profesores de Educación Física de Almería (APEF) y Federación Española de Asociaciones de Docentes de Educación Física (FEADEF)

Abraldes, JA., Rodríguez, N., Rubio, JA. y Martínez, P. (2006). Actualización y aprendizaje de la RCP Básica. Coruña: Federación de Salvamento e Socorrismo de Galicia.

Axelsson, A., Herlitz, J. y Fridlund, B. (2000). How bystanders perceive their cardiopulmonary resuscitation intervention; a qualitative study. Resuscitation, 47(1), 71-81

Celenza, T., Gennat, HC., O’Brien, D., Jacobs, IG., Lynch, DM. y Jelinek, GA. (2002). Community competence in cardiopulmonary resuscitation. Resuscitation, 55(2), 157-165.

Cummins, RO., Ornato, JP., Thies, WH. y Pepe, PE. (1991). Improving survival from sudden cardiac arrest: the «chain of survival» concept. A statement for health professionals from the Advanced Cardiac Life Support Subcommittee and the Emergency Cardiac Care Committee, American Heart Association. Circulation, 83(5), 18321847.

Eisenburger, P. y Safar, P. (1999). Life supporting first aid training of the public-review and recommendations. Resuscitation, 41(1), 3-18.

European Resuscitation Council.(ERC). (2005). Recomendaciones para la reanimación 2005 del European Resuscitation Council. Resuscitation, $67 \mathrm{~s} 1,1-44$.
Fernández Balboa, JM. (1997): La investigación en la Educación Física española: un índice para el futuro. Apunts. Educación Física y Deportes, 50, 100-106.

Frederick, K., Bixby, E., Orzel, MN., Stewart-Brown, S. y Willett, K. (2002). Will changing the emphasis from 'pulseless' to 'no signs of circulation' improve the recall scores for effective life support skills in children? Resuscitation, 55(3), 255-261.

García, N., Solís, V. y Vásquez, S. (1998). Grado de conocimiento de profesores de educación general básica sobre primeros auxilios en traumatismos del aparato locomotor. Tesis doctoral presentada en la Universidad Católica de Chile. Sin Publicar.

Gómez, J. (1990): Metodología de encuesta por muestreo. En J. Arnau, MT. Anguera y J. Gómez. Metodología de la investigación en ciencias del comportamiento. (pp. 237-310). Murcia: Universidad de Murcia.

Hernández, R., Fernández, C. y Baptista, P. (2000). Metodología de la investigación. México: Mc Graw Hill.

INE. (2006). Defunciones según la causa de muerte. Año 2004. Notas de prensa.

Lafferty, C., Larsen, P. D. y Galletly, D. (2003). Resuscitation teaching in New Zealand schools. N Z Med J, 116(1181), U582.

Lewis, RM., Fulstow, R. y Smith, GB. (1997). The teaching of cardiopulmonary resuscitation in schools in Hampshire. Resuscitation, 35(1), 27-31.

Liberman, M., Golberg, N., Mulder, D. y Sampalis, J. (2000). Teaching cardiopulmonary resuscitation to CEGEP students in Quebec - a pilot project. Resuscitation, 47(3), 249-257.

Magalhães, M. y Hill, A. (2005). Investigação por questionário. Lisboa: Edições Silabo.

Moreira, JM. (2004). Questionários: Teoria e práctica. Coimbra: Almedina.

Morgan, CL., Donnelly, PD., Lester, CA. y Assar, DH. (1996). Effectiveness of the BBC's 999 training roadshows on cardiopulmonary resuscitation: video performance of cohort of unforewarned participants at home six months afterwards. Bmj, 313(7062), 912-916.

Ortín, A. y Abraldes, JA. (2007). Analysis of knowledge in first aid of

physical education teachers of secondary education in relation to the type of centre in which they works. En JA. Abraldes y N. Rodríguez. (Eds.). Book of abstracts. International Lifesaving Congress. Pp. 126. La Coruña: Federación de Salvamento e Socorrismo de Galicia.

Pearn, J. (2000). Basic life support: extending and integrating teaching in the Australian community. Aust N Z J Surg, 70(1), 3-5.

Sekimoto, M., Noguchi, Y., Rahman, M., Hira, K., Fukui, M., Enzan, K., et al. (2001). Estimating the effect of bystander-initiated cardiopulmonary resuscitation in Japan. Resuscitation, 50(2), 153160.

Selby, ML., Kautz, JA., Moore, TJ., Gombeski, WR., Ramirez, AG, Farge, EJ., et al. (1982). Indicators of response to a mass media CPR recruitment campaign. Am J Public Health, 72(9), 1039-1042.

Uray, T., Lunzer, A., Ochsenhofer, A., Thanikkel, L., Zingerle, R., Lillie, P., et al. (2003). Feasibility of life-supporting first-aid (LSFA) training as a mandatory subject in primary schools. Resuscitation, 59(2), 211-220.

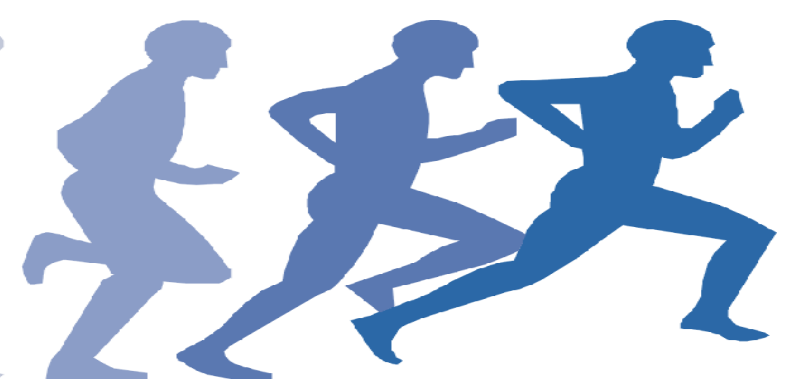


